Article

\title{
Secondary Metabolite Localization by Autofluorescence in Living Plant Cells
}

\author{
Pascale Talamond ${ }^{1, *}$, Jean-Luc Verdeil ${ }^{2}$ and Geneviève Conéjéro ${ }^{2}$ \\ 1 Institut des Sciences de l'Evolution Montpellier ISE-M, Université Montpellier, CNRS, IRD, \\ EPHE, CC 065, Place Eugène Bataillon, 34095 Montpellier, France \\ 2 Histocytology and Plant Cell Imaging platform PHIV, UMR AGAP (CIRAD, INRA, SupAgro)-UMR \\ B\&PMP (INRA, CNRS, SupAgro, Montpellier University), 34095 Montpellier, France; \\ E-Mails: verdeil@cirad.fr(J.-L.V.); genevieve.conejero@supagro.inra.fr (G.C.) \\ * Author to whom correspondence should be addressed; E-Mail: pascale.talamond@ird.fr; \\ Tel.: +33-467-14-42-37; Fax: +33-467-14-36-22.
}

Academic Editor: Kevin D. Belfield

Received: 3 June 2014 / Accepted: 25 February 2015 / Published: 19 March 2015

\begin{abstract}
Autofluorescent molecules are abundant in plant cells and spectral images offer means for analyzing their spectra, yielding information on their accumulation and function. Based on their fluorescence characteristics, an imaging approach using multiphoton microscopy was designed to assess localization of the endogenous fluorophores in living plant cells. This method, which requires no previous treatment, provides an effective experimental tool for discriminating between multiple naturally-occurring fluorophores in living-tissues. Combined with advanced Linear Unmixing, the spectral analysis extends the possibilities and enables the simultaneous detection of fluorescent molecules reliably separating overlapping emission spectra. However, as with any technology, the possibility for artifactual results does exist. This methodological article presents an overview of the applications of tissular and intra-cellular localization of these intrinsic fluorophores in leaves and fruits (here for coffee and vanilla). This method will provide new opportunities for studying cellular environments and the behavior of endogenous fluorophores in the intracellular environment.
\end{abstract}

Keywords: autofluorescence; secondary metabolites; phenolic compounds; hydroxycinnamic acids; plant tissue; spectral imaging; Linear Unmixing 


\section{Introduction}

When excited by UV/Vis radiation of suitable wavelengths, we can observe the fluorescence emission from endogenous fluorescent molecules in living tissue (algae, plant and animal). This fluorescence emission, called autofluorescence, is an intrinsic property of cells. Nevertheless, one must be able to distinguish these intrinsic signals from other fluorescent signals resulting from the addition of exogenous markers [1]. Although the intensity of autofluorescent markers is quite low, it can interfere with the signals of the exogenous fluorescent markers used in typical spectral imaging experiments. In plant tissues, the extracellular matrix and cellular components often contribute to autofluorescence emission to a greater extent than exogenous fluorescent markers [2]. Fluorescence imaging in green plants is unusually challenging because of the large amounts of intracellular fluorophore molecules [3]. Chlorophyll, the main photosynthetic pigment of plants, absorbs the majority of light and emits a strong fluorescence. Nevertheless, this potentially annoying property can be used as an advantage in spectral imaging for obtaining information on their localization and functions on living plants.

Advanced research in imaging spectral microscopy has focused on increasing the discrimination between distinct fluorophores with highly overlapping emission spectra and thus enhance the possibilities of multicolor imaging [1,4]. After no less than one decade of development, imaging method using spectral analysis coupled with advanced Linear Unmixing has become a vital biological imaging tool that combines the advantages of optical imaging and spectroscopy $[1,2]$. This label-free imaging capability of microscopy methods can reveal autofluorescence emissions from the endogenous fluorophores in living cells and tissues [5]. We developed an approach in imaging spectral coupled to Linear Unmixing which has to ability to gather of spectral profiles about endogenous flurorophores enabling the detection and differentiation of mixed fluorophores in green plant tissues.

UV excitation (with wavelengths from 340 to $360 \mathrm{~nm}$ ) of green leaves generates two types of fluorescence: a blue-green fluorescence and red and far-red fluorescence [3,6,7]. Altogether, the red and far-red fluorescence of plant cells are called "chlorophyll fluorescence" because they correspond to the sole intrinsic fluorophore in plant leaves with emission in this part of spectrum. The blue-green fluorescence has long been identified, but it has not fostered much investigation and it has not been systematically studied until recently. Unlike the red fluorescence, which is almost all derived from chlorophyll, the blue fluorescence of plants combines the emissions of several universal cellular flurophores [8,9] including: nicotinamide (NADH), flavin (FMN, FAD) coenzymes, pyridoxal phosphate (vitamine $\mathrm{B}_{6}$ ), folic acid (vitamin $\mathrm{B}_{9}$ ) and secondary metabolites including the three main groups (phenolics, alkaloids and terpenoids). This fluorescence is characterized by a wide spectral emission band in the blue band (430-450 nm) and a shoulder in the green band (520-530 nm) [6]. From an intact leaf, the detected fluorescence is emitted mainly by compounds located in the walls of epidermal cells and vascular bundles [10-12].

Secondary metabolites refer to a generic term used for more than 200,000 different substances that are exclusively found in plant and sometimes in fungi [13]. Plants produce a vast array of secondary metabolites in response to interactions with their environment, which impart flavor, color, bitterness, astringency and fragrance, and confer protection through a variety of antimicrobial, pesticidal and pharmacological properties. Some flavonoids and coumarins (chromones), pterines (pyridoxal 
coenzyme), some phenols (hydroxycinnamoyl acids) and some alkaloids (caffeine) in plant emit in the blue region (450-500 nm), flavins and some terpenoids in the green region (500-530 nm), while polyacetylene, isoquinoline and some alkaloids emit in the yellow and orange regions of the visible spectrum and anthocyanins and anthocyanidins emit in the red region of the visible spectrum [14-17] (Figure 1).

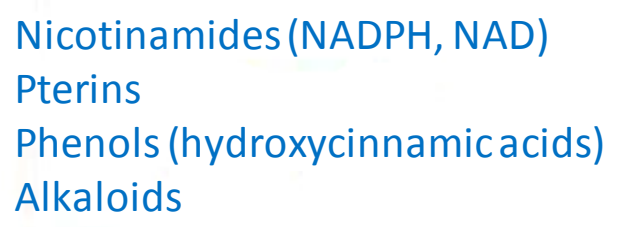

Figure 1. Fluorescence emission spectrum of a typical green leaf under UV-radiation $\left(\lambda_{\text {exc. }}=355 \mathrm{~nm}\right)$. The fluorescence bands in blue $(430-450 \mathrm{~nm})$, green $(520-530 \mathrm{~nm})$, red (680), and far-red (740 $\mathrm{nm})$ regions, adapted from [6].

Metabolites and enzymes involved in the biosynthesis of phenylpropanoids, terpenoids and alkaloids have been localized in a variety of plant cells. Methods for detecting the tissue-specific distribution of phenolic compounds have been developed by coupling microspectrofluorometry and multispectral fluorescence microimaging or by confocal laser scanning microscopy [18-20]. These methods enabled the discrimination of the relative abundance of hydroxycinnamic derivates and flavonoids in different layers of plant leaves. However, the cell type-specific localization of most secondary metabolic pathways has not yet been characterized. Several physiological functions have been hypothesized, but little is known about the biosynthesis and metabolism of these bioactive compounds in plants.

Here, the performance of these methods was demonstrated for plants, focusing on leaf and fruit tissues, i.e., the adaxial and abaxial epidermis, and palisade and spongy mesophyll. Developments in microscopic techniques, such as confocal laser scanning microscopy, provide a unique opportunity for studying tissue localization of phenolic compounds more precisely than with conventional fluorescence microscopy [21]. Furthermore, multiphoton microscopy outperforms confocal microscopy, due to its deeper tissue penetration and reduced phototoxicity [22]. Here, we describe how this method allowed a finer discrimination of the relative abundances of secondary metabolites in particular, phenolic compounds in different layers of tissues, namely: (1) chlorogenic acid (5-caffeoylquinic acid, 5-CQA)), mangiferin (C2- $\beta$-D-glucoside-1,3,6,7-tetrahydroxyxanthen-9-one) and caffeine 
(1,3,7-trimethylxanthine) in coffee leaves and (2) vanillin glucoside (4- $O$-(3-methoxybenzldehyde)- $\beta$ D-glucoside) in vanilla fruit.

\section{Results and Discussion}

\subsection{Autofluorescence of Living Plant Cells and Tissues}

Endogenous fluorophores are particularly abundant in plant tissues and are involved in structural and metabolic functions at cell and tissues level $[8,9,23]$. Several studies have been performed, both in vivo and in vitro, on the use of autofluorescence-based techniques, in particular studies with fluorescence microscopy, for the discrimination the tissues. The basic fluorescence microscope irradiates the tissues with a desired and specific band of wavelength and then to separate the much weaker emitted fluorescence (Figure 2).
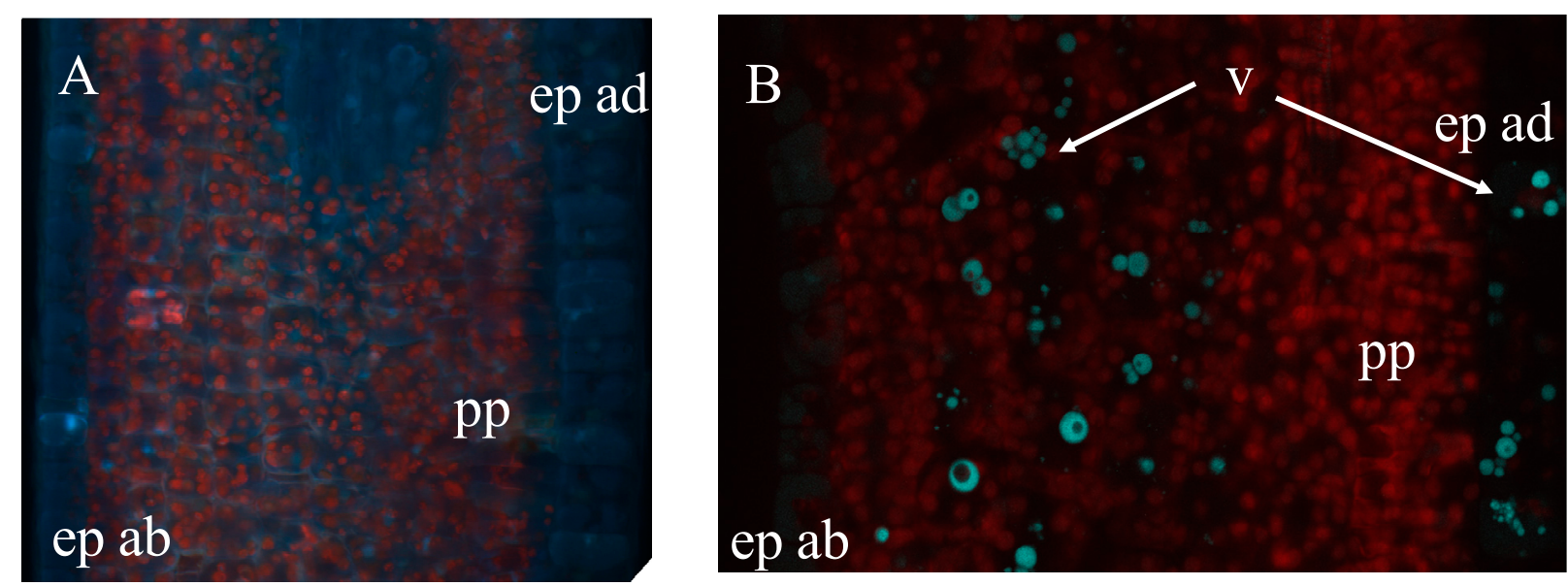

Figure 2. Autofluorescence of fresh young leaf in a cross-section of Coffea canephora observed with a epifluorescence microscope (A, filter exc: 340-380 nm, em: 425-800 nm) and with a multiphoton microscope (B, exc: laser IR $720 \mathrm{~nm}$, em: channel 435-485 nm in blue, channel 670-700 nm in red) ep: epidermis, pp: palisade parenchyma, v: vesicle.

A cross-section of a live young leaf of Coffea canephora observed with an epifluorescence miscroscope was found to emit two types of autofluorescence under UV light at 340-380 nm: (1) a red fluorescence of chlorophyll in chloroplast, originating from the palisade parenchyma cells; and (2) a white-blue fluorescence originating from some vesicles and vacuoles in the mesophyll cells and the epidermis (Figure 2A). The same sample observed with a multiphoton microscope at $720 \mathrm{~nm}$ excitation (UV-like excitation light) gave a better resolution to identify the subcellular anatomy (Figure 2B) and avoided the phototoxicity of the UV lamp usually used with a wide-field microscope. The relative intensities of this blue fluorescence are highly sensitive and species-specific and they further depend on environmental factors $[8,14]$. The fluorescence emission spectrum induced by UV radiation in a particular plant can be considered as a molecular signature that reflects environmental (biotic or abiotic) effects and can reveal the significance of secondary metabolites. In this region of the UV spectrum, the intracellular fluorophores that are most often encountered are universal in plants such as pyridinic and pterine coenzymes, pyrodoxal-5'-phosphate (PLP) and some phenolic compounds 
and alkaloids. Hydroxycinnamic acid esters (HQA) and xanthine derivates are the principal secondary metabolites found in the leaves of commercial coffee trees. In young leaves, the HQA esters represent $0.3 \%$ to $7.8 \%$ of the dry weight depending on plant species [24,25]. In C. Canephora leaves, hydroxycinnamic acid esters are the main secondary metabolites and they could be responsible for the absorption maximum around $450 \mathrm{~nm}$ [8,26,27], as other fluorophores absorbing at other frequencies are less abundant. In order to interpret this pattern; it is crucial to understand the respective contributions of every fluorophore to the autofluorescence signature.

\subsection{Spectral Signatures of Endogenous Fluorophores}

The relatively high-transmission spectral region between $\sim 400-800 \mathrm{~nm}$ is often described as the "optical window" where biological tissues both absorb and scatter light quite strongly. Cells contain molecules that become fluorescent when excited by visual radiation of suitable wavelength. This autofluorescence emission, by endogenous fluorophores is an intrinsic property; therefore the spectrum provides an accurate fingerprint of these molecules. In two-photon microscopy-pulsed ( $\sim 100$ femtoseconds) infra-red laser light is used to excite fluorophores that can absorb visible light $[28,29]$ Excitation is restricted to a very tiny volume in the sample (one femtoliter). The laser intensity in the focal volume is so high- that each fluorophore absorbs two photons, in contrast to conventional fluorescence where a single photon is absorbed. With two photon excitation, only the focal volume is excited and the out-of-focus volume is unaffected [30]. Laser light, in the range 700 to $1050 \mathrm{~nm}$, is scattered to a much lesser extent than visible and UV wavelengths, it penetrates at least twice deeper into plant tissues $(\sim 500 \mu \mathrm{m})$ and thus allows for 3D imaging of thicker specimens [28]. In addition, the combined energies of two low-energy photons are equivalent to the energy of one high-energy photon. Single-photon spectra (absorption, emission) are well documented for a wide range of molecules [31]. However, the increasing use of non-linear miscroscopy, which relies on multiphoton technology, requires knowledge of multiphoton photophysics and especially the two-photon excitation properties of fluorophores and biological molecules. It can be presumed that these spectra do not differ from those of single-photon spectra, but experimental evidences are lacking. Therefore, experiments were conducted to ascertain that the spectral characteristics of single and two-photon excited autofluorescence were identical.

Conventional spectroscopy was achieved using pulsed Xenon light source. The two-photon emission spectral signatures were obtained by spectral acquisition $\lambda_{\mathrm{exc}}=720 \mathrm{~nm}$. The detection bandwidth was set to collect emissions from 365 to $700 \mathrm{~nm}$, using a specific PMT (photomultiplier tube) detector. The spectral characteristics of single-photon excited fluorescence (SPEF) and two-photon excited fluorescence (TPEF) signals were obtained for chlorophyll(a), chlorogenic acid, caffeine, mangiferin and vanillin 4-O- $\beta$-D-glucoside, which are the major fluorophores in coffee leaves (C. canephora, C. pseudozanguebariae) and vanilla fruits (Vanilla planifolia Jackson ex Andrews), respectively, and are shown in Table 1 . The absorption spectra with two-photons are much broader than those with a single-photon. This could explain the red shift in emission maximum. The excitation wavelengths were selected as a function of the available laser equipment and were as close as possible to the optimal excitation wavelengths of the fluorophores under study. With TPEF at $720 \mathrm{~nm}$, the excitation wavelength is too distant from optimal and the fluorescence intensity of caffeine is at least 
100 times weaker than at the optimal wavelength of $272 \mathrm{~nm}$ (SPEF). The full absorption and emission spectra of chlorogenic acid were acquired in the spectral range between 250 and $600 \mathrm{~nm}$ (Figure 3).

Table 1. Spectral regions of absorption and emission maxima with conventional spectrometry and multiphoton laser for five endogenous fluorophores.

\begin{tabular}{ccccc}
\hline \multirow{2}{*}{ Metabolite } & \multicolumn{2}{c}{ Conventional Spectrometer } & & Multiphoton Laser \\
\cline { 2 - 3 } & $\begin{array}{c}\boldsymbol{\lambda}_{\text {absorption }} \\
\text { Maxima (nm) }\end{array}$ & $\begin{array}{c}\boldsymbol{\lambda}_{\text {emission }} \\
\text { Maxima/Bandwidth (nm) }\end{array}$ & & $\begin{array}{c}\boldsymbol{\lambda}_{\text {emission }} \\
\text { Maxima/Bandwidth (nm) * }\end{array}$ \\
\hline Chlorophyll a & 430 & $685 / 22$ & $660 / 35$ \\
Chlorogenic acid & 324 & $455 / 90$ & $453 / 105$ \\
Methoxybenzaldehyde & 318 & $424 / 90$ & $515 / 115$ \\
Mangiferin & 318,366 & nd & $570 / 80$ \\
Caffeine & 272 & $397 / 80$ & $405 / 60$ \\
\hline
\end{tabular}

$* \lambda_{\max } /$ bandwidth of fluorescence emission that is measured by the width of spectral profile at 50 percent of the maximum yield.
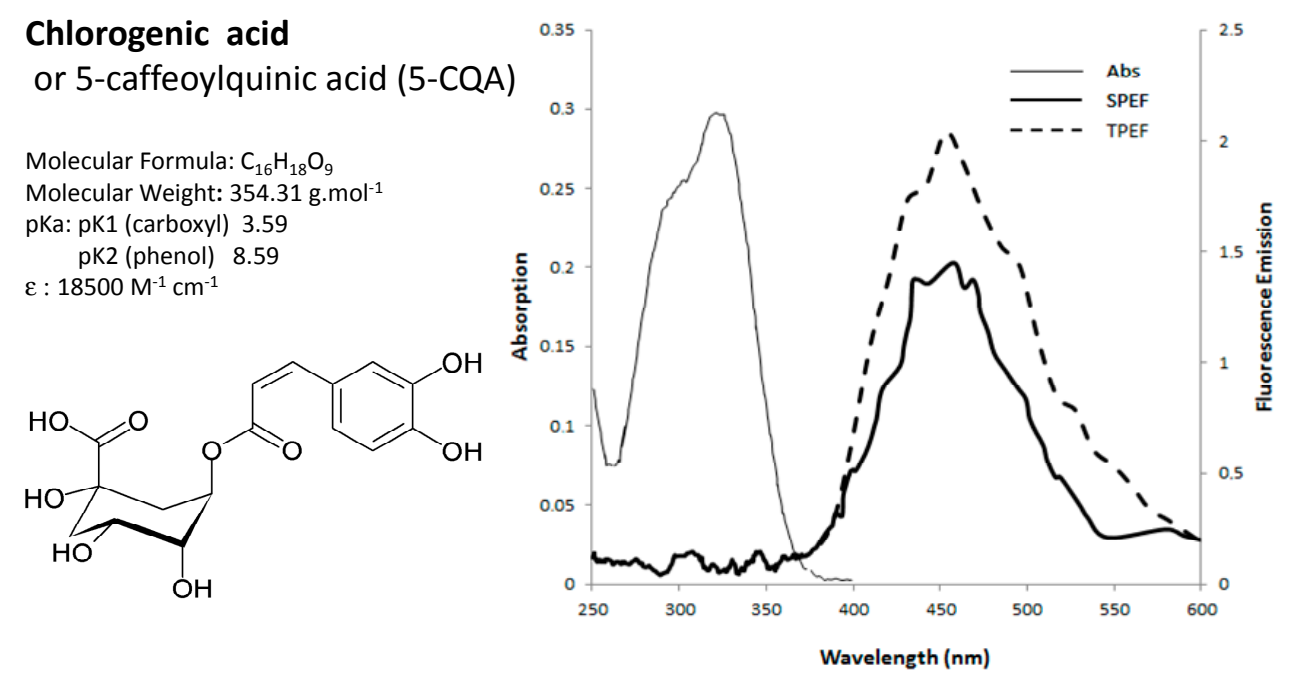

Figure 3. Absorption and emission spectra of chlorogenic acid (5-caffeoylquinic acid, 5-CQA) with conventional spectrometer (single photon excited fluorescence, SPEF) and multiphoton laser (two-photon excited fluorescence, TPEF) and the chemical characteristics.

No differences between SPEF and TPEF emission spectra were found for the peak $\lambda_{\max }$ and bandwidth of four endogenous fluorophores: chlorophyll, chlorogenic acid, mangiferin and caffeine. By contrast, the SPEF and TPEF signals of vanillin 4-O- $\beta$-D-glucoside excited at $300 \mathrm{~nm}$ and $720 \mathrm{~nm}$ exhibited about $90 \mathrm{~nm}$ redshift and bandwidth broader compared with the SPEF spectra. In general SPEF and TPEF spectra are identical (e.g., [32]) but some studies found a redshift between the SPEF and TPEF spectra, for example for 7-hydroxycoumarine [33], another phenolic compound, and for pure coenzyme fluorophore (NADH) [34]. The reasons behind these spectral differences remain to be elucidated. 


\subsection{Phenolic Compounds Localization in Coffee Leaf}

Using a multiphoton microscope set at a $720 \mathrm{~nm}$ excitation wavelenght, Linear Unmixing generated the image illustrated in Figure 4. This method uses an array of photomultiplier tubes and a polychromatic multichannel detector between 360 and $700 \mathrm{~nm}$. The Linear Unmixing method compares the measured spectrum, which can be composed of multiple fluorescent components to a library of reference spectra obtained from the analysis of pure compounds. We fitted the measured emission signal at each image pixel with the function $\mathrm{I}(\lambda)$ sum, computed according to the equation in given in Section 3. A composite image was generated from four base images, two of which corresponding to the two pure (reference) compounds, and the third one to the residual fluorescence channel (Figure 4C,D). Linear Unmixing separates the summed spectral data into individual images for each reference fluorophore, by using the least square difference principle applied at the pixel level [35].
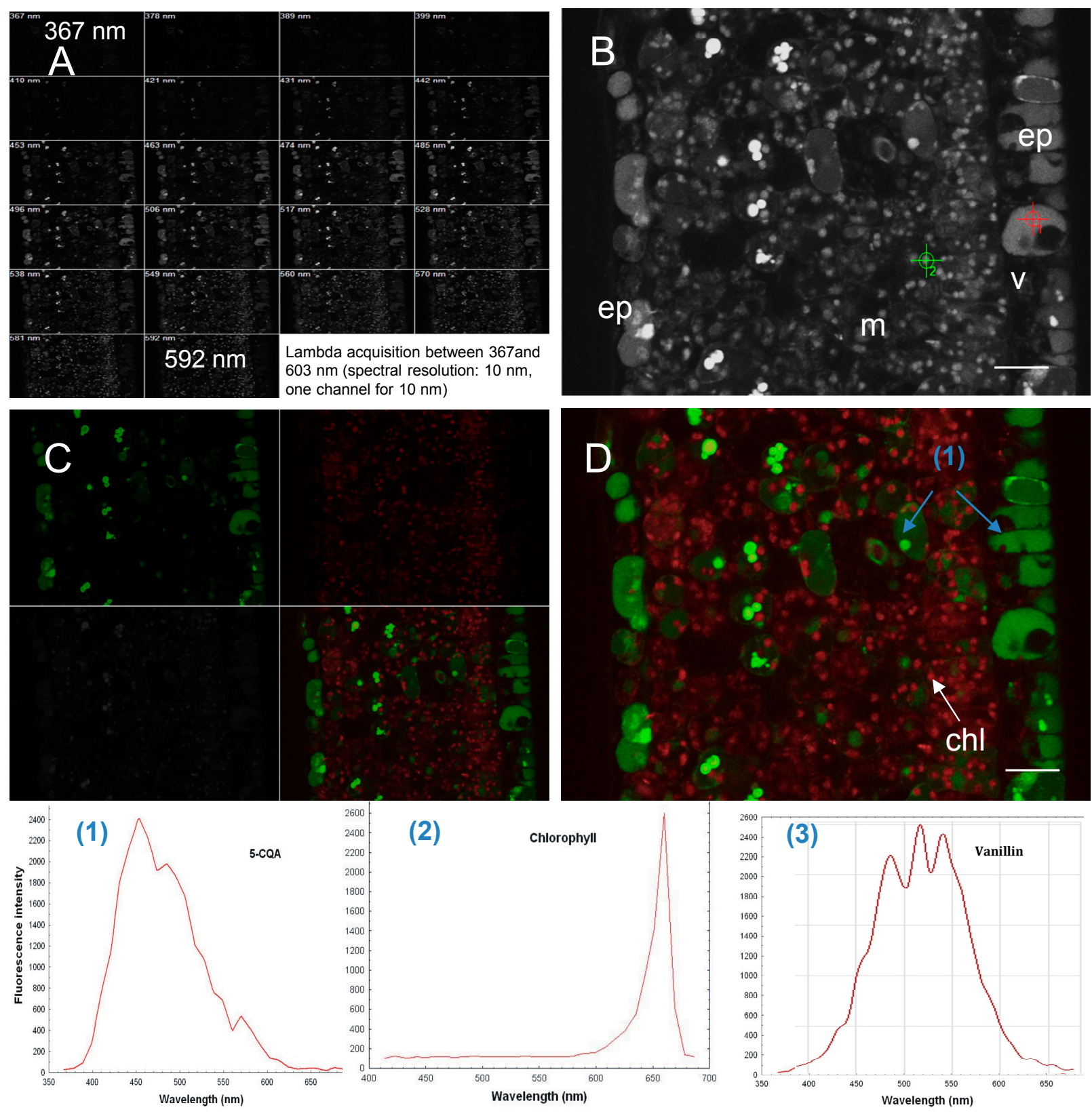

Figure 4. Cont. 

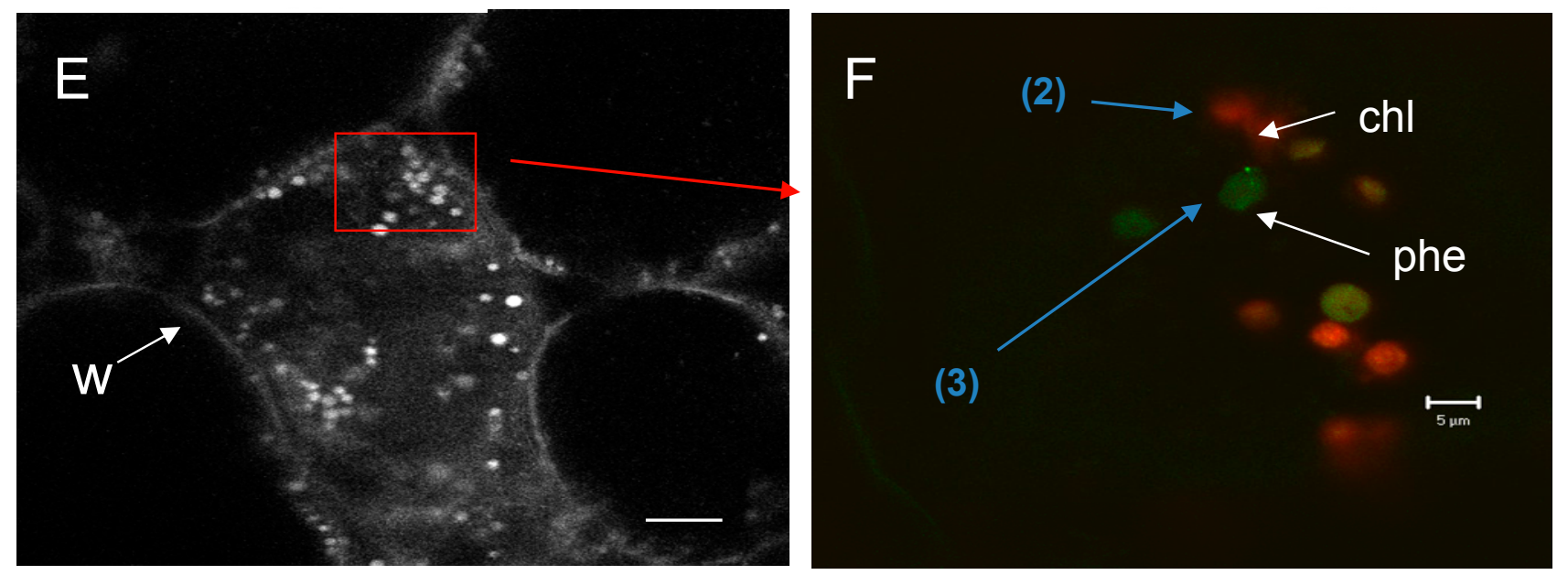

Figure 4. Spectral imaging and analysis in cross sections of Coffea canephora young leaf and Vanilla planifolia fruit by multiphotonic microscopy, lambda excitation: $720 \mathrm{~nm}$. (A) Images gallery obtained after lambda acquisition between 367 and $603 \mathrm{~nm}$ (spectral resolution: $10 \mathrm{~nm}$, one channel for $10 \mathrm{~nm}$ ); (B) Spectral acquisition, overlay of different channels of A, named Lambda Max; (C) Linear Unmixing, (left: split, right: overlay of 3 channels); (D) Linear Unmixing with emission spectra of chorophyll (red) and 5CQA or chlorogenic acid (green) in Coffea canephora young leaf: overlay image of three channels and corresponding to fourth image of C; (E) Spectral acquisition (F) Linear Unmixing with emission spectra of 4-O-(3- methoxybenzaldehyde)- $\beta$-D-glucoside (green) and chlorophyll (red) in inner placenta cells of Vanilla planifolia pods. The spectral images are false color. Reference emission spectra of 5CQA (1), chlorophyll (2) and vanillin 4-O- $\beta$-D-glucoside (3). (chl, chloroplast; ep, epidermis; m, mesophyll; phe, phenyloplast; w, cell wall; v, vacuol). Scale bar: A and B: $20 \mu \mathrm{m}$; C: $50 \mu \mathrm{m}$; D: $5 \mu \mathrm{m}$.

The spectral signature is determined for each pixel of the scanned image and is used for the subsequent digital separation of fluorescent molecules. Then, the Linear Unmixing process enables the discrimination of between various fluorescence signals, even between those with widely overlapping emission spectra. Reference spectral signatures were acquired on the microscope using controls of standard powders known to be present in a $C$. canephora and C. pseudozanguebariae leaf blade cross-section. Each control molecule (in the case of coffee leaves, chlorogenic acid, caffeine, mangiferin and chlorophyll spectrum) is carried out with the same excitation wavelength (720 nm) and exactly the same optical conditions. The spectral signature was specific for every molecule and stored in the Spectra Database.

Since reference $\lambda$-stacks are determined by the spectral properties of the respective fluorophores and are independent of their concentrations, they can be used to assemble a matrix fluorophore with specific weighting factors, which is subsequently used to determine the respective contributions of each fluorophore to each image pixel [1]. The sensitivity of the method depends directly on the sensitivity of the detector and spectral characteristics of the fluorophores under study. The spatial resolution is determined by pixel size (here $\sim 0.4 \mu \mathrm{m}^{2}$ ) under our experimental conditions. Caffeine is not detected in epidermal cells because its fluorescence intensity is too weak for this particular excitation wavelength and too far away from its nominal wavelength. Chlorogenic acid (5-CQA) and 
mangiferin both exhibited a broad spectral range with peaks at about 425 and $520 \mathrm{~nm}$, respectively [27]. Fluorophores occur mainly in epidermis cells and particularly in vesicles (Figure 4B).

Mangiferin fluorescence appeared in small vesicles of the parenchyma and in vacuoles of the abaxial epidermis, but not in the adaxial epidermis [27]. By contrast, 5-CQA fluorescence was abundant in the abaxial epidermis and constituted an important reservoir of phenolic compounds, but its role has not been clearly defined yet. In sun leaves of Phillyrea latifolia, flavonoids largely accumulated in the adaxial epidermal layer and subepidermal cells [19]. The localization of these phenolic compounds in the vacuoles of epidermal cells, and in the vesicles and chloroplasts of mesophylic cells further supports the hypothesis of their involvement in leaf protection against UV damages or pathogens [20,26,27].

To date, phenolic compound histolocalization methods mainly uses the standard reagent for phenols and flavonoids, the Neu's reagent, and then examination by epifluorescence microscopy [36,37]. Excitation with UV laser beam at $364 \mathrm{~nm}$ induced strong green fluorescence in the epidermal cells of coffee leaves, which was attributed to the presence of phenolic compounds [24]. In juvenile leaf blades, an intense greenish-white fluorescence was observed in all chlorenchyma cells, including the palisade and spongy mesophyll cells [24]. Under UV-light, in absence of any interference with the intrinsic red fluorescence of chlorophyll, detection of phenolic compounds and derivatives from hydroxycinnamic acid esters, in particular ferrulic and caffeic acids, was possible. Another phenolic compound, the mangiferin ( $C$-glucosylxanthone), a yellow-pigment found in wild coffee species does not require any reagent and was directly observed though autofluorescence [38,39]. The young leaves of wild coffee species (Coffea pseudozanguebariae) showed a yellow and orange fluorescence under UV light [39]. In a typical spectral imaging, the potential of spectral microscopy has been broadly demonstrated by the use of emission spectral signature in living plant cells marked different fluorophores. There are usually several fluorophores present in sample not to determine the relative contribution from each endogenous fluorophores. Our approach is different and novel because our method detects only on a base of this specific spectral analysis for discriminating between multiple endogenous fluorophores.

\subsection{Phenolic Compound in Vanilla Fruit}

As was the case for coffee, the histolocalisation of phenolic compounds in vanilla fruit with a Schiff's reagent produced a fuschia stain, indicating that they were filled with an aldehyde-bearing substance. This response enables no accurate distinction between various aldehydes and is not informative as whether theses aldehydes differ from glycosylated 4-O-(3-methoxybenzldehyde) form. The autofluorescence was characterized to localize and 4-O-(3-methoxybenzaldehyde)- $\beta$-D-glucoside and chlorophyll in vanilla fruit. The aforementioned procedure was applied to inner mesocarpal cells using two reference spectra, i.e., the responses of chlorophyll and 4-O-(3-methoxybenzldehyde)- $\beta$-D-glucoside excited at $740 \mathrm{~nm}$ [40]. Linear Unmixing was applied by using the reference spectra and deducing the flurophores at the pixel's level. The result of the Linear Unmixing showed that the content of some like-chloroplast organelles in vanilla fruit was 4-O-(3-methoxybenzldehyde)- $\beta$-D-glucoside, while some others contained both only chlorophyll, or both chlorophyll and this glucoside (Figure 4E,F). Thus, it appears that the storage of 4-O-(3-methoxybenzldehyde)- $\beta$-D-glucoside varies between chloroplasts 
in the inner mesocarp of vanilla fruit. Spectral analysis combined with biochemical data and immunocytological and electron microscopy studies allowed us to identify a new chloroplast-derived organelle, the phenyloplast, that stores an important phenolic compound [40].

Spectral analysis provides a number of important advantages: it allows a molecule to be tracked in the living material by limiting artifacts related to sample preparation. Unlike the situation with histochemical staining reagents, it can locate fluorophore by its specific spectral signature. Spectral imaging and Linear Unmixing extends the possibilities of obtaining specific strong and sustainable signals without degradation of cellular functions in living tissue.

\section{Experimental Section}

\subsection{General Experimental Procedures}

Epi-fluorescence microscopy was carried out on a Leica DM 6000 instrument equipped with a Q-Imaging camera (long-pass filter $425 \mathrm{~nm}$, QImaging, Surrey, BC, Canada). A Zeiss 510 META NLO multiphoton microscope (Zeiss, Jena, Germany) equipped with a Coherent Chameleon Ultra II laser was used to obtain emission fluorescence from fresh leaves. A 25×/0.8 Plan-Neofluar immersion objective was used to obtain galleries of spectral images and emission spectra from fresh plant sections. The multiphoton microscope with tunable laser Chameleon Ultra II (690-1080 nm range excitation, Coherent, Santa Clara, CA, USA) enables the excitation of secondary metabolites in a manner similar to an UV laser. Optimal excitation was obtained at $\lambda=720 \mathrm{~nm}$ and band-pass emission in the 365-700 $\mathrm{nm}$ range using an array of 32 photomultiplier tube (PMT) detectors (Zeiss), each with a $10.7 \mathrm{~nm}$ bandwidth. This procedure was performed on live tissue cross-sections, as well as on pure standards for: chlorogenic acid (5-CQA, 5 caffeoyl-quinic acid), caffeine (1,3,7,-trimethylxanthine) (Sigma-Aldrich, St Quentin Fallavier, France), mangiferin (C2- $\beta$-D-glucoside-1,3,6,7-tetrahydroxyxanthen9-one) (Extrasynthese, Genay, France), and 4-O-(3-methoxybenzaldehyde) $\beta$-D-glucoside (Sigma-Aldrich) powders. The chlorogenic acid (5-CQA) in a methanol solution in was measured on a FL-2500 fluorescence spectrophotometer (Hitachi, Tokyo, Japan). The excitation wavelength was $320 \mathrm{~nm}$ and the emission wavelength was within the $250-600 \mathrm{~nm}$ range.

Spectral analysis was carried out using the advanced Linear Unmixing function (LSM 510 software, Zeiss) which that separates mixed signals pixel by pixel using the entire emission spectrum of each defined autofluorescent compound in the sample. This function requires at least two spectra and was applied with the advanced iterative option and one residual channel. After spectral imaging acquisitions on leaf cross-sections, the advanced Linear Unmixing function allowed visualization with coded colours of the fluorescence of each standard and chlorophyll in cell based on their reference spectra.

\subsection{Processing Spectral Images}

The fundamental concept underlying Linear Unmixing calculations is each pixel in the spectral image is categorized as representing a mixture of fluorophore signals (intensities) when the measured spectrum $(\mathrm{I}(\lambda))$ can be deconvolved into the proportion, weight, or concentration $(\mathrm{C})$ of each individual fluorophore reference spectrum $(R(\lambda))$ when the values are summed. Thus, each reference spectrum of 
a pure fluorophore is described as $\operatorname{Ri}(\lambda)$ where $i=1,2,3 \ldots . . n$ represents the index of the fluorophore (Ci). For a particular number of fluorophores (n), this relationship can be represented as [35]:

$$
\begin{gathered}
\mathrm{I}(\lambda)=\mathrm{C} 1 \mathrm{xR} 1(\lambda)+\mathrm{C} 2 \mathrm{xR} 2(\lambda)+\mathrm{C} 3 \times \mathrm{R} 3(\lambda)+\ldots \ldots .+\mathrm{Cn} \times \mathrm{Rn}(\lambda) \\
\text { Or more simply: } \\
\mathrm{I}(\lambda)=\sum \mathrm{i} \mathrm{Ci} \times \operatorname{Ri}(\lambda)
\end{gathered}
$$

In practice, the signal intensity for each pixel (I) in the spectral image is determined and recorded during acquisition of the lambda stack and the reference spectra for the known fluorophores are measured independently in separate control specimens labelled with only a single fluorophore, using identical sample preparation techniques and instrument settings. The overall spectral contributions from the various fluorophores in the specimen can then be determined as a simple linear algebra matrix exercise by calculating their individual contributions to each point in the measured spectrum, as described in the equations above. For many of Linear Unmixing software packages, the solution is obtained by inputting reference spectral profiles and using an inverse least squares fitting approach that minimizes the square difference between the measured and the calculated spectra.

\subsection{Plant Material}

In these experiments we used Coffea plants from the collection cultivated at the IRD Research Centre, in Montpellier, France. Leaves of C. Canephora (DB56, DB57) and C. pseudozanguebariae $(\mathrm{H} 65, \mathrm{H} 70)$ of various genotypes were taken from trees maintained under tropical conditions (natural daylight, $25{ }^{\circ} \mathrm{C}, 28{ }^{\circ} \mathrm{C}, 78 \%-82 \%$ humidity). Vanilla (Vanilla planifolia Jackson ex Andrews) from the Réunion Island, France, was hand-pollinated. Sound fruits were harvested 4 months and 7 months after pollination and air-freighted in a refrigerated box and delivered to laboratory within 2 days after hand-picking.

\subsection{Leaf and Fruit Cross-Sections}

Leaf and fruit cross-sections $(50 \mu \mathrm{m})$ were obtained using HM $650 \mathrm{~V}$ vibrating blade microtome (Microm, Waldorf, Germany). Cross-sections were dipped in $0.1 \mathrm{M}$ phosphate buffer salin with ascorbic acid to prevent oxidation.

\section{Conclusions}

Visualization of secondary metabolites of plant by spectral analysis of their autofluorescence under UV-like laser is a novel approach to their localization [26,27,40,41]. The autofluorescence of endogenous fluorophores is used to enable their localization in plant cells and tissues. This was achieved with a special detection system, using spectral analysis, coupled to advanced Linear Unmixing method. This method enables the discrimination of various fluorescence signals, even with widely overlapping emission spectra. The ability to carry out measurements on live tissues without any preparation treatment, such as fixation or staining makes autofluorescence-based techniques of utmost interest for obtaining information on cell morphology, behavior, and metabolism [42]. This approach requires a good knowledge of anatomy of sample and must be correlated with biochemical data about 
studied molecules. Thereby, the potential for this approach to improve our understanding of bimolecular interaction dynamics is huge and allowing to explore new biology questions not only in plants.

\section{Acknowledgments}

The authors are grateful for financial support from the Conseil Régional of Languedoc-Roussillon (PRI) and the French Institute for the Development (IRD) and thank Etienne Baras for help in reviewing the English language.

\section{Author Contributions}

Geneviève Conéjéro and Pascale Talamond have contributed in performing experiments, to the chemical and physical analysis of samples and Jean-Luc Verdeil analyzed the data. All authors participated in drafting the manuscript; they have read and approved the final manuscript.

\section{Conflicts of Interest}

The authors declare there is no conflict of interest.

\section{References}

1. Zimmerman, T.; Rietdorf, J.; Pepperkok, R. Spectral imaging and its application in live cell microscopy. FEBS Lett. 2003, 546, 87-92.

2. Berg, R.H. Evaluation of spectral imaging for plant cell analysis. J. Microsc. 2004, 214, 174-181.

3. Chappelle, E.W.; Wood, F.M.; McMurtrey, J.E.L.; Newcomb, W.W. Laser induced fluorescence of green plants. 1: A technique for the remote detection of plant stress and species differentiation. Appl. Opt. 1984, 23, 134-138.

4. Tsurui, H.; Nishimura, H.; Hattori, S.; Hirose, S.; Okumura, K.; Shirai, T. Seven-color fluorescence imaging of tissue samples based on Fourier spectroscopy and singular value decomposition. J. Histochem. Cytochem. 2000, 48, 653-662.

5. Mylle, E.; Codreanu, M.C.; Boruc, J.; Russinova, E. Emission spectra profiling of fluorescent proteins in living plant cells. Plant Methods 2013, 9, 1-8.

6. Buschmann, C.; Langsdorf, G.; Lichtenthaler, H.K. Imaging of blue, green and red fluorescence emission of plants: On overview. Photosynthetica 2000, 38, 483-491.

7. Meyer, G.E.; Cartelat, A.; Moya, L.; Cerovic, Z.G. UV-induced blue-green and farred fluorescence along wheat leaves: A potential signature of leaf ageing. J. Exp. Bot. 2003, 54, 757-769.

8. Cerovic, Z.G.; Samson, G.; Morales, F.; Trembaly, N.; Moya, I. Ultraviolet-induced fluorescence for plant monitoring present state and prospects. Agronomie 1999, 19, 543-578.

9. Monici, M. Cell and tissue autofluorescence research and diagnostic applications. Biotechnol. Annu. Rev. 2005, 11, 1387-2656.

10. Chappelle, E.W.; Wood, F.M.; McMurtray, J.E.I.; Newcomb, W.W. Laser induced fluorescence of green plants. 3: LIF spectral signatures of five major plant types. Appl. Opt. 1985, 24, 74-80.

11. Haris, P.J.; Hartley, R.D. Detection of bound ferulic acid in the cell walls of the Gramineae by ultra-violet fluorescence microscopy. Nature 1980, 259, 508-510. 
12. Ounis, A.; Cerovic, Z.G.; Briantais, J.M.; Moya, I. Dual-excitation FLIDAR for the estimation of epidermal UV absorption in leaves and canopies. Remote Sens. Environ. 2001, 76, 33-48.

13. Hartmann, T. From waste products to ecochemicals: Fifty years research of plant secondary metabolism. Phytochemistry 2007, 68, 2831-2846.

14. Buschmann, C.; Lichtenthaler, H.K. Principles and characteristics of multicolour fluorescence imaging of plants. J. Plant Physiol. 1998, 152, 297-314.

15. Wolfbeis, O.S. The fluorescence of organic natural products, chap 3. In Molecular Luminescence Spectroscopy: Methods and Applications; Schulman, S.G., Ed.; John Wiley \& Sons: New York, NY, USA, 1985; Volume 77, pp. 167-370.

16. Roshchina, V.V. Fluorescing World of Plant Secreting Cells; BIOS Scientific Publishers Limited: Milton Park, UK, 2007.

17. Roshchina, V.V. Vital Autofluorescence: Application to the study of plant living cells. Int. J. Spectrosc. 2012, 2012, doi:10.1155/2012/124672.

18. Agati, G. Response of the in vivo chlorophyll fluorescence spectrum to environmental factors and laser excitation wavelength. Pure Appl. Opt. 1998, 7, 797-807.

19. Agati, G.; Galardi, C.; Gravano, E.; Romani, A.; Tattini, M. Flavonoid Distribution in Tissues of Phillyrea latifolia L. Leaves as Estimated by Microspectrofluorometry and Multispectral Fluorescence Microimaging. Photochem. Photobiol. 2002, 76, 350-360.

20. Tattini, M.; Galardi, C.; Pinelli, P.; Massai, R.; Remorini, D.; Agati, G. Differential accumulation of flavonoids and hydroxycinnamates in leaves of Ligustrum vulgare under excess light and dought stress. New Phytol. 2004, 163, 547-561.

21. Hutzler, P.; Fischbach, R.; Heller, W. Tissue localization of phenolic compounds in plants by confocal laser scanning microscopy. J. Exp. Bot. 1998, 49, 953-965.

22. Denk, W.; Strickler, J.; Webb, W. Two-photon laser scanning fluorescence microscopy. Science 1990, 248, 73-76.

23. Rost, F.W.D. Autofluorecence in plants, fungi and bacteria. In Fluorescence Microscopy; Rost, F.W.D., Ed.; Cambridge University Press: Cambridge, UK, 1995; Volume 2, pp. 16-36.

24. Mondolot, L.; la Fisca, P.; Buatois, B.; Talansier, E.; de Kochko, A.; Campa, C. Evolution in caffeoylquinic acid content and histolocalization during Coffea canephora leaf development. Ann. Bot. 2006, 98, 33-40.

25. Campa, C.; Mondolot, L.; Rakotondravao, A.; Bidel, L.P.; Gargadennec, A.; Couturon, E.; la Fisca, P.; Rakotomalala, J.J.; Jay-Allemand, C.; Davis, A.P. A survey of mangiferin and hydroxycinnamic acid ester accumulation in coffee (Coffea) leaves: Biological implications and uses. Ann Bot. 2012, 110, 595-613.

26. Conejero, G.; Talamond, P.; Verdeil, J.L. A new approach to visualize secondary metabolites in plants. In Proceedings of the FESPB, XVII Congress of Federation of European Societies of Plant Biology, Valencia, Spain, 4-9 July 2010.

27. Conejero, G.; Noirot, M.; Talamond, P.; Verdeil, J.L. Spectral analysis combined with advanced linear unmixing allows for histolocalization of phenolics in leaves of coffee trees. Front. Plant Sci. 2014, 5, 39-46.

28. Rubart, M. Two-Photon Microscopy of Cells and Tissue. Circ. Res. 2004, 95, 1154-1165. 
29. Xu, C.; Zipfel, W.; Shear, J.B.; Williams, R.M.; Webb, W.W. Multiphoton fluorescence excitation: New spectral windows for biological nonlinear microscopy. Proc. Natl. Acad. Sci. USA 1996, 93, 10763-10768.

30. Patterson, G.H.; Piston, D.W. Photobleaching in Two-Photon Excitation Microscopy. Biophys. J. 2000, 78, 2159-2162.

31. Smith, W.L. Handbook of Laser Science and Technology, supplement 2; Weber, M.J., Ed.; CRC Press (Chemical Rubber Comapgny): Baca Raton, FL, USA, 1986; pp. 229-258.

32. Xu, C.; Webb, W.W. Measurement of two-photon excitation cross sections of molecular fluorophores with data from to $1050 \mathrm{~nm}$. J. Opt. Soc. Am. B 1996, 13, 481-491.

33. Parma, L.; Omenetto, N. Fluorescence behavior of 7-hydroxycoumarine excited by one-photon and two-photon absorption by means of a tunable dye laser. Chem. Phys. Lett. 1978, 54, 544-546.

34. Bestvater, F.; Spiess, E.; Stobrawa, G.; Hacker, M.; Feurer, T.; Porwol, T.; Berchner-Pfannschmidt, U.; Wotzlaw, C.; Acher, H. Two-photon fluorescence absorption and emission spectra of dyes relavant for cell imaging. J. Microsc. 2002, 208, 108-115.

35. Garini, Y.; Young, I.T.; McNamara, G. Spectral imaging: Principles and Applications. Cytometry Part A 2006, 69, 735-747.

36. Neu, R. A new reagent for differentiating and determining flavones on paper chromatograms. Naturwissenschaften 1957, 43, 82, doi:10.1007/BF00631858.

37. Dai, G.H.; Andary, C.; Mondolot-Cosson, L.; Boubals, D. Histochemical responses of leaves of in vitro plantlets of Vitis spp. to infection with Plasmopara viticola. Phytopathology 1995, 85, 149-154.

38. Talamond, P.; Mondolot, L.; Gardennec, A.; de Kochko, A.; Hamon, S.; Fruchier, A.; Campa, C. First report on mangiferin ( $C$-glucosyl-xanthone) isolated from leaves of a wild coffee plant, Coffea pseudozanguebariae (Rubiaceae). Acta Bot. Gallica 2008, 155, 513-519.

39. Talamond, P.; Conéjéro, G.; Verdeil, J.L.; Poëssel, J.L. Isolation of $C$-glycosyl Xanthones from Coffea pseudozanguebariae and their Location. Nat. Prod. Commun. 2011, 6, 1885-1888.

40. Brillouet, J.M.; Verdeil, J.L.; Odoux, E.; Lartaud, M.; Grisoni, M.; Conéjéro, G. Phenol homeostasis is ensured in vanilla fruit by storage under solid form in a new chloroplast-derived organelle, the phenyloplast. J. Exp. Bot. 2014, 65, 2427-2435.

41. Brillouet, J.M.; Romieu, C.; Schoefs, B.; Solymosi, K.; Cheynier, V.; Fulcrand, H.; Verdeil, J.L.; Conéjéro, G. The tannosome is an organelle forming condensed tannins in the chlorophyllous organs of Tracheophyta. Ann. Bot. 2013, 112, 1003-1014.

42. Littlejohn, G.R.; Meckel, T.; Scharzländer, M.; Costa, A. Functional imaging in living plants-cell biology meets physiology. Front. Plant Sci. 2014, 5, 1-3.

Sample Availability: Samples of the compounds are not available from the authors.

(C) 2015 by the authors; licensee MDPI, Basel, Switzerland. This article is an open access article distributed under the terms and conditions of the Creative Commons Attribution license (http://creativecommons.org/licenses/by/4.0/). 